13

\title{
The effect of pretilt and twisted angle on twisted nematic liquid crystal filter
}

\author{
(C) Han Hua, Yang Liu, Kong Yong
}

School of Electrical and Electronic Engineering, Shanghai University of Engineering Science, Shanghai 201620, China

e-mail: kkyy7757@163.com

Received July 24, 2017

The effect of pretilt and twisted angle on twisted nematic liquid crystal filter (TNLCF) is studied theoretically and experimentally in this paper, based on the birefringence and distortion characteristic of twisted nematic liquid crystal, the output performance of TNLCF has been analyzed through numerical simulation firstly, then the corresponding experiment results verify the correctness of the simulation results, which show that the output performance of TNLCF is indeed related to pretilt angle and twisted angle of liquid crystal, with the increasing of the twisted angle, the central wavelength of TNLCF will shift to the long wavelength, with the increasing of pretilt angle for the top glass substrate and the decreasing of pretilt angle for the bottom glass substrate, the central wavelength of TNLCF will shift to the short wavelength and if the pretilt angle of the bottom glass substrate is increased and the pretilt angle of the top glass substrate is reduced at the same time in some certain value or otherwise, the shift trend of the central wavelength will be not evident. These results will offer an important reference value for the design and application of TNLCF as we believe.

DOI: $10.21883 /$ OS.2018.08.46369.224-17

* Полный текст статьи опубликован в английской версии журнала. 